\title{
POTENCIAL ENERGÉTICO DOS RESÍDUOS DO FRUTO DA MACAÚBA E SUA UTILIZAÇÃO NA PRODUÇÃO DE CARVÃO VEGETAL
}

\author{
ENERGY POTENTIAL OF THE MACAW PALM FRUIT RESIDUES AND THEIR USE IN \\ CHARCOAL PRODUCTION
}

\author{
Anderson Barbosa Evaristo $^{1}$ Daniela Correia Martino ${ }^{2}$ Adriano Henrique Ferrarez ${ }^{3}$ \\ Danilo Barros Donato ${ }^{4}$ Angélica de Cássia Oliveira Carneiro ${ }^{5}$ José Antônio Saraiva Grossi ${ }^{6}$
}

\begin{abstract}
RESUMO
O uso da biomassa para fins energéticos tem sido destaque como forma de reduzir a dependência energética de fontes não renováveis. A macaúba, espécie Acrocomia aculeata (Jacq.) Lodd. ex Martius, é uma palmeira arborescente. Possui um grande potencial energético a partir do processamento de seus frutos. O objetivo desse trabalho foi caracterizar os resíduos da macaúba após o processamento dos frutos, a fim de avaliar a sua utilização in natura e como carvão vegetal. O endocarpo apresentou teor de carbono fixo, poder calorífico e densidade a granel superiores, quando comparado aos demais resíduos e menores teores de cinza. O poder calorífico do carvão do endocarpo foi superior, enquanto que os teores de materiais voláteis do carvão do epicarpo apresentaram os maiores resultados. Todos os resíduos avaliados do processamento do fruto da macaúba têm potencial na geração de energia, especialmente o endocarpo e o epicarpo. $O$ carvão do endocarpo possui características adequadas para sua utilização como fonte de energia e como redutor.

Palavras-chave: Acrocomia aculeata; bioenergia; macaúba.
\end{abstract}

\begin{abstract}
The use of biomass for energy purposes has been highlighted in as a way to reduce dependence on nonrenewable energy sources. Macaw palm, Acrocomia aculeata (Jacq.) Lodd. ex Martius, is an arborescent palm and has a high energy potential from the fruit processing. The aim of this study was to characterize the macaw palm residues after the fruit processing to evaluate its use "in natura" and as charcoal. The endocarp showed higher fixed carbon content, heating value and bulk density compared with other residues and lower ashes content. The heating value of endocarp charcoal was the highest, whereas the volatile matter levels of epicarp charcoal showed higher results. All evaluated residues from the macaw palm fruit processing have potential in power generation, especially the endocarp and the epicarp. The endocarp charcoal has suitable characteristics as source of energy and as a reductant.
\end{abstract}

Keywords: Acrocomia aculeata; bioenergy; macaw palm.

1 Engenheiro Agrônomo, Dsc., Professor do Curso de Engenharia Agronômica, Fundação Universidade do Tocantins - Unitnis, 108 Sul Alameda 11 Lote 03, CEP: 77020-122, Palmas (TO), Brasil. evaristo.ab@gmail.com

2 Engenheira Florestal, MSc, Doutorando em Ciência Florestal pela Universidade Federal de Viçosa, Av. P.H. Rolfs, Campus Universitário, CEP 36570-000, Viçosa (MG), Brasil. danimartinodem@gmail.com

3 Físico, Professor do Instituto Federal Fluminense no Campus Itaperuna, Doutorando em Engenharia Agrícola, Universidade Federal de Viçosa, Av. P.H. Rolfs, Campus Universitário, CEP 36570-000, Viçosa (MG), Brasil. adriano.ferrarez@ufv.br

4 Engenheiro Florestal, MSc, Doutorando em Ciência Florestal pela Universidade Federal de Viçosa, Av. P.H. Rolfs, Campus Universitário, CEP 36570-000, Viçosa (MG), Brasil. danilobarros20@hotmail.com

5 Engenheira Florestal, $\mathrm{Dr}^{\mathrm{a}}$, Professora do Departamento de Engenharia Florestal, Universidade Federal de Viçosa, Av. P.H. Rolfs, Campus Universitário, CEP 36570-000, Viçosa (MG), Brasil. cassiacarneiro@ufv.br

6 Engenheiro Agrônomo, PhD, Professor do Departamento de Fitotecnia, Universidade Federal de Viçosa, Av. P.H. Rolfs, Campus Universitário, CEP 36570-000, Viçosa (MG), Brasil. jgrossi@ufv.br

Recebido para publicação em 16/08/2013 e aceito em 12/08/2014 


\section{INTRODUÇÃO}

O futuro da energia no mundo é uma das grandes preocupações atuais da humanidade. Dentro deste contexto, as energias renováveis se tornam solução no enfrentamento das mudanças climáticas e na diminuição da dependência das fontes fósseis de energia (HIRSCHL, 2009). Neste sentido, a biomassa tem despertado interesse para fins energéticos, em virtude de seu grande potencial de aproveitamento.

Em muitos países, principalmente os de clima tropical, há uma enorme quantidade de resíduos produzidos anualmente provenientes dos setores agrícola e florestal. Grande parte destas biomassas vem sendo utilizada no meio rural com baixa eficiência energética na produção de energia elétrica ou na produção de calor para secagem de produtos agrícolas (SILVA; SOBRINHO; SAIKI, 2004). A transformação dos resíduos em biocombustíveis é uma alternativa interessante, uma vez que proporciona um ganho no rendimento energético, qualidade e redução dos custos de transportes. Hoje há diversas tecnologias disponíveis para conversão de resíduos em biocombustíveis, tendo um destaque em nível nacional o carvão vegetal. O Brasil é o maior produtor de carvão vegetal do mundo, com 48,6 milhões de toneladas e representa $14 \%$ da produção mundial (ORGANIZAÇÃO DAS NAÇÕES UNIDAS PARA ALIMENTAÇÃO E AGRICULTURA, 2011). Sua utilização tem sido principalmente nas indústrias siderúrgica, cimento, alimentícia e química. No país, cerca de $80 \%$ do consumo de carvão vegetal é destinado aos setores de ferro-gusa, ferro-liga e aço (EMPRESA DE PESQUISA ENERGÉTICA, 2011). Neste cenário, o Estado de Minas Gerais destaca-se atendendo $60 \%$ da demanda de carvão vegetal do Brasil.

Várias matérias-primas têm sido indicadas para diversos tipos de biocombustíveis nos últimos anos. Atualmente, têm se destacado as espécies sacarinas, oleaginosas e essências florestais como culturas energéticas para produção de energia. Uma espécie que tem despertando muito interesse como matéria-prima na produção de biocombustíveis é a palmeira macaúba devido ao seu alto teor de óleo no fruto, produção de diversos resíduos energéticos além de ser uma planta perene, nativa e encontrada em diversos biomas do território brasileiro.

A macaúba, espécie Acrocomia aculeata (Jacq.) Lodd. ex Martius, é uma palmeira arborescente, espinhosa, que pode ultrapassar $16 \mathrm{~m}$ de altura e está presente ao longo de toda a América tropical e subtropical, do sul do México ao sul do Brasil, não ocorrendo apenas no Equador e Peru (HENDERSON et al., 1995; SCARIOT; LLERAS; HAY, 1991; 1995). Possui grande potencial para produção de óleos que pode ser aplicado nos setores industrial e energético. Além da grande produção de óleo, possui outras vantagens em relação às demais oleaginosas como os resíduos gerados com o processamento, no qual tem um grande potencial energético o que pode implicar na redução de custos de produção e geração de receita para a unidade de beneficiamento (LORENZI, et al., 2004; MIRANDA et al., 2001; ROLIM, 1981). O fruto da macaúba é esférico, tipo drupa, sendo constituído pela casca fibrosa (epicarpo), polpa oleaginosa (mesocarpo), endocarpo e amêndoa oleaginosa. Dentre estas partes constituintes, o endocarpo, em especial, pode ser convertido em carvão vegetal, constituindose em um subproduto da cadeia produtiva do biodiesel, podendo ser utilizado na metalurgia, siderurgia ou para cocção (FUNDAÇÃO CENTRO TECNOLÓGICO DE MINAS GERAIS, 1983).

O objetivo desse trabalho foi caracterizar os resíduos da macaúba após o processamento dos frutos com a finalidade de avaliar a sua utilização na forma in natura e de carvão vegetal. Foram avaliadas as propriedades químicas e físicas dos resíduos e da carbonização do epicarpo e endocarpo, além dos rendimentos gravimétricos do carvão destes resíduos.

\section{MATERIAL E MÉTODO}

Foram coletados frutos da palmeira macaúba, idade desconhecida, de uma população de plantas da fazenda experimental da EPAMIG, Empresa de Pesquisa Agropecuária de Minas Gerais, localizado no município de Prudente de Morais, Minas Gerais. Os frutos fisiologicamente maduros foram destacados dos cachos, selecionados e homogeneizados.

Para determinação da porcentagem da massa úmida e seca, foram selecionadas quatro amostras contendo 20 frutos por amostra. Estes frutos foram processados e separados de suas partes constituintes (epicarpo, mesocarpo, endocarpo e amêndoa). Posteriormente, procedeu-se à secagem de cada constituinte do fruto para a determinação da porcentagem de massa seca e úmida. Inicialmente, cada amostra foi pesada, obtendo-se assim sua massa úmida, e logo em seguida estas foram levadas 
em estufa e secas a $75^{\circ} \mathrm{C}$. Após atingirem massa constante, as amostras foram novamente pesadas, determinando-se assim sua massa seca.

Os resíduos foram formados a partir do processamento dos frutos, que constituiu em retirada das cascas, prensagem do mesocarpo (polpa) e amêndoa e separação do endocarpo. O mesocarpo e amêndoa foram prensados em uma prensa hidráulica para extração do óleo e em seguida os seus resíduos foram lavados em um extrator de óleos e graxas para retirada do óleo residual, utilizandose o n-hexano como solvente orgânico. Após esta etapa, todos os resíduos do processamento dos frutos (casca, mesocarpo, endocarpo e amêndoa), foram moídos e peneirados a uma granulometria de aproximadamente $0,2 \mathrm{~mm}$.

Para a análise química imediata, amostras dos resíduos foram secas em estufa, pesando-se 1,0 g de amostra absolutamente seca (a.s.) em uma cápsula de porcelana, utilizando para isto uma balança de precisão. Posteriormente, as cápsulas foram tampadas e levadas em forno mufla a $950 \pm 10^{\circ} \mathrm{C}$ durante 11 minutos. $\mathrm{O}$ material restante na cápsula sem a tampa, após a determinação do percentual de materiais voláteis, foi utilizado para determinar o percentual de cinzas, a uma temperatura de 600 $\pm 10^{\circ} \mathrm{C}$ durante 6 horas. $\mathrm{O}$ percentual de carbono fixo (CF) foi determinado por diferença entre a soma do percentual de materiais voláteis, cinzas e 100 , conforme os procedimentos recomendados na norma ABNT NBR 8112 (1986).

$\mathrm{O}$ poder calorífico superior (PCS) dos resíduos do processamento do fruto da macaúba foram determinados de acordo com a metodologia descrita pela norma da ABNT NBR 8633 (1984), utilizando-se uma bomba calorimétrica adiabática.

A densidade a granel (DG) foi determinada de acordo com a norma estabelecida pela DIN EM 15103 (2010), para as partes do fruto (epicarpo, mesocarpo, endocarpo e amêndoa). Colocaram-se as partes em uma caixa rígida de volume conhecido e logo em seguida essas foram pesadas. Tanto para o mesocarpo quanto para a amêndoa foi determinada a densidade a granel destas partes sem óleo (farelo da polpa e amêndoa).

A densidade energética dos resíduos da macaúba foi calculada de acordo com a equação abaixo:

$$
\mathrm{DE}=\mathrm{PCS} X \mathrm{DG}
$$

Em que: $\mathrm{DE}=$ densidade Energética; $\mathrm{PCS}=$ poder calorífico superior; $\mathrm{DG}=$ densidade a granel.
As carbonizações foram realizadas no forno mufla de laboratório com aquecimento elétrico, utilizando-se cerca de $500 \mathrm{~g}$ de amostras de cada constituinte (endocarpo e epicarpo), os quais foram inseridos em um container metálico com dimensões nominais de $30 \mathrm{~cm}$ de comprimento e $12 \mathrm{~cm}$ de diâmetro. A temperatura inicial e final de carbonização foi de 100 e $550^{\circ} \mathrm{C}$, respectivamente, sendo que a cada 30 minutos de carbonização aumentava-se a temperatura do forno em $50^{\circ} \mathrm{C}$, finalizando o processo em quatro horas e meia. Foram recuperados os gases condensáveis do processo por um condensador tubular resfriado a água, adaptado na saída dos gases. Ao final de cada carbonização procedeu à pesagem do carvão e dos produtos condensados determinando assim com base na massa seca dos resíduos, os rendimentos gravimétricos em carvão vegetal, gases condensáveis e não condensáveis, sendo que o resultado do valor deste último foi obtido por diferença.

Após as carbonizações, foram realizadas as análises química e física do carvão vegetal. Foi determinada a composição química imediata do carvão vegetal, sendo que esta foi obtida em amostras moídas e peneiradas a uma granulometria de, aproximadamente, $0,2 \mathrm{~mm}$, seguindo os procedimentos preconizados nas normas ABNT NBR 6923 (1981) e ABNT NBR 8112 (1986), com determinação dos teores de matérias voláteis, cinzas e carbono fixo, em base seca.

O poder calorífico superior do carvão vegetal, foi determinado de acordo com o mesmo método descrito acima utilizado para os resíduos.

Já a densidade aparente do carvão vegetal, foi determinada de acordo com o método proposto por Vital (1984), utilizando-se uma balança de precisão de $0,001 \mathrm{~g}$ para a determinação do volume deslocado. Para tanto, amostras de aproximadamente $2 \mathrm{~g}$ de carvão foram pesadas para a obtenção da massa e, posteriormente, imersas em mercúrio para determinação do volume deslocado, determinando assim a densidade relativa aparente (DA). A densidade energética para o carvão vegetal foi dada pela multiplicação da DA pelo PCS. Todas as análises e as carbonizações foram realizadas no Laboratório de Painéis e Energia da Madeira (LAPEM) da Universidade Federal de Viçosa (UFV).

O experimento foi instalado no delineamento inteiramente casualizado (DIC) com três repetições, quando os tratamentos eram os resíduos e duas repetições quando os tratamentos eram o carvão 
do endocarpo e epicarpo. Já as análises dos constituintes em que foram submetidos ao processo de carbonização também foram feitas de acordo com um DIC com dois tratamentos. Para as análises de química imediata utilizaram-se três repetições e para o poder calorífico superior duas repetições. As análises de variância foram processadas no DIC e as diferenças entre as médias dos tratamentos foram analisadas pelo teste $\mathrm{F}$ e Tukey, a $5 \%$ de probabilidade. Todas as análises foram processadas no programa SAS, versão 9.0.

\section{RESULTADOS E DISCUSSÃO}

A porcentagem de massa úmida e seca e o peso médio dos frutos da macaúba estão apresentados na Tabela 1.

A parte constituinte de maior proporção do fruto é a polpa seguida pelo epicarpo, endocarpo e amêndoa. Em relação à massa seca, o endocarpo representa a segunda parte de maior abundância no fruto.

A polpa representa a maior proporção em relação ao fruto devido ao seu maior teor de água e seu elevado teor de óleo. Ciconini et al. (2013), estudando a biometria dos frutos e o teor de óleo, encontraram nas populações de macaúba do cerrado do Mato Grosso do Sul, 49,1\% e 25,1\% teor de umidade e de óleo, respectivamente. Cetec, (1983) estudando as populações de macaúba em Minas Gerais encontrou teor de óleo na polpa superior, 55-69\%, e verificou, também, que a amêndoa apesar de sua baixa proporção em relação ao fruto contém também alto teor de óleo, $55-58 \%$, o que proporciona baixa produção de resíduo para utilização energética.

$\mathrm{Na}$ Tabela 2 são apresentados os valores médios das propriedades físicas e químicas dos resíduos da macaúba para energia.

Observa-se que houve diferença significativa em todos os parâmetros avaliados, evidenciando as diferenças existentes entre as partes do fruto da macaúba. Os maiores teores de materiais voláteis foram obtidos nos resíduos da polpa e da amêndoa, enquanto que o endocarpo apresentou os menores teores de cinzas e os maiores teores de carbono fixo.

Os teores de materiais voláteis dos resíduos da macaúba estão próximos aos normalmente obtidos para a maioria da biomassa vegetal, que está compreendida entre 65 a $83 \%$. Ressalta-se que o percentual de material volátil é importante principalmente para a ignição e nas etapas iniciais da combustão da biomassa (CORTEZ; LORA, 2008). Em relação às cinzas, a diferença entre seus

TABELA 1: Massa úmida, massa seca e peso fresco das partes do fruto de macaúba.

TABLE 1: Wet weight, dry weight and fresh weight of macaw palm fruit parts.

\begin{tabular}{lccccc}
\hline \multicolumn{1}{c}{ Parâmetro } & Epicarpo & Polpa & Endocarpo & Amêndoa & Fruto \\
\hline Massa Úmida (\%) & $26,69^{1} \pm 0,46$ & $45,78 \pm 0,59$ & $22,39 \pm 0,31$ & $5,14 \pm 0,26$ & 100 \\
Massa Seca (\%) & $22,2 \pm 0,55$ & $38,48 \pm 1,02$ & $32,00 \pm 0,43$ & $7,32 \pm 0,23$ & 100 \\
Peso fresco (g) & $11,11 \pm 0,57$ & $19,05 \pm 0,61$ & $9,32 \pm 0,45$ & $2,14 \pm 0,11$ & $41,01 \pm 1,64$ \\
\hline
\end{tabular}

Em que: ${ }^{1}$ Média e desvio padrão

TABELA 2: Propriedades físicas e químicas dos resíduos do processamento dos frutos da macaúba. Densidade a granel (DG), poder calorífico superior (PCS) e densidade energética (DE).

TABLE 2: Physical and chemical properties of the macaw palm fruits processing residues. Bulk density (BD), heating value (HV) and energy density (ED).

\begin{tabular}{lcccccc}
\hline \multicolumn{1}{c}{ Resíduo } & $\begin{array}{c}\text { Materiais } \\
\text { Voláteis }(\%)\end{array}$ & $\begin{array}{c}\text { Cinzas } \\
(\%)\end{array}$ & $\begin{array}{c}\text { Carbono fixo } \\
(\%)\end{array}$ & $\begin{array}{c}\text { DG } \\
\left(\mathrm{Kg} / \mathrm{m}^{3}\right)\end{array}$ & PCS $(\mathrm{Kcal} / \mathrm{kg})$ & $\mathrm{DE}\left(\mathrm{GJ} / \mathrm{m}^{3}\right)$ \\
\hline Epicarpo & $78,22 \mathrm{ab}$ & $5,66 \mathrm{~b}$ & $16,12 \mathrm{~b}$ & $177 \mathrm{~d}$ & $4989 \mathrm{a}$ & $3,64 \mathrm{c}$ \\
Farelo da polpa & $79,6 \mathrm{a}$ & $6,19 \mathrm{c}$ & $14,20 \mathrm{~b}$ & $338 \mathrm{~b}$ & $3835 \mathrm{c}$ & $5,43 \mathrm{~b}$ \\
Endocarpo & $76,37 \mathrm{~b}$ & $2,04 \mathrm{a}$ & $21,33 \mathrm{a}$ & $498 \mathrm{a}$ & $5011 \mathrm{a}$ & $10,45 \mathrm{a}$ \\
Farelo da amêndoa & $78,9 \mathrm{a}$ & $6,2 \mathrm{c}$ & $14,9 \mathrm{~b}$ & $287 \mathrm{c}$ & $4377 \mathrm{~b}$ & $5,26 \mathrm{~b}$ \\
\hline
\end{tabular}

Em que: Médias seguidas pela mesma letra na coluna não diferem entre si, a $1 \%$ de probabilidade pelo teste Tukey $5 \%$. 
teores está provavelmente na constituição mineral de cada resíduo. Ramos et al. (2008) encontraram na polpa de macaúba valores médios de $61,96,36,70$ e 766,37mg.100 $\mathrm{gr}^{-1}$ de cálcio, fósforo e potássio, respectivamente. CETEC (1983) observou valores de cinzas similares nos resíduos dos frutos e altos teores de fósforo e potássio nas cinzas da amêndoa e polpa, respectivamente.

Quanto às propriedades físicas, observa-se que o endocarpo se destaca por apresentar maiores valores médios de densidade a granel, poder calorífico superior e densidade energética em relação aos demais. Embora o epicarpo possuísse alto PCS, observa-se que o mesmo apresentou a menor DE, devido a sua baixa DG quando comparado aos outros resíduos. Provavelmente, o alto valor de PCS tanto do endocarpo quanto do epicarpo se deve à alta concentração de carbono fixo e altos teores de lignina (parâmetro não mensurado). Estudos da CETEC (1983) mostraram que o epicarpo apresenta em média $29,5 \%$ de lignina e a polpa, endocarpo e amêndoa possuem em torno de 7,9, 34 e 10,2\% de lignina, respectivamente. Outro fator que contribui para o alto PCS são resquícios de óleo da polpa aderido no epicarpo durante o processamento (avaliação visual). Boas et al. (2010) estudando o efeito da temperatura de carbonização sobre resíduos de macaúba, também encontraram PCS do endocarpo, $5.152 \mathrm{Kcal}_{\mathrm{kg}}{ }^{-1}$, semelhante ao obtido neste trabalho. Tanto o poder calorifico quanto o carbono fixo dos resíduos do processamento dos frutos da macaúba são superiores ou próximos a diversos resíduos agrícolas e madeireiros utilizados para produção de energia, evidenciando o potencial dos resíduos da macaúba para geração de energia. Paula et al. (2011) trabalhando com diversos resíduos de diversas culturas agrícolas (arroz, café, cana-de-açúcar, soja, feijão e milho) e com resíduos do setor madeireiro (aparas e serragem) encontraram PCS variando de 3.812 a $4.615 \mathrm{Kcal} / \mathrm{kg}$ e carbono fixo de 16,66 a $21,03 \%$.

Por ser um material mais lignificado e denso, o endocarpo possui maior densidade a granel e aliado ao seu alto PCS, apresentou-se como o resíduo de maior densidade energética comparado com os demais. Materiais com baixa densidade energética são obstáculos para sua utilização, uma vez que podem aumentar consideravelmente os custos de transportes e afetar negativamente o balanço energético do sistema.

$\mathrm{Na}$ Tabela 3 são apresentadas as propriedades físicas e químicas do carvão vegetal produzido a partir do endocarpo e epicarpo da macaúba.

Observa-se que houve efeito do tipo de resíduo sobre as propriedades avaliadas, exceto densidade a granel.

Observa-se que a exceção dos teores de cinzas e materiais voláteis, as demais propriedades do carvão do endocarpo foram superiores ao do carvão do epicarpo.

Houve acréscimo no PCS, CF, DE e a redução dos materiais voláteis do carvão do endocarpo e epicarpo em relação às suas respectivas matérias-primas. Este acréscimo do $\mathrm{CF}$ e redução dos voláteis são devido às altas temperaturas dentro do forno, uma vez que durante a carbonização ocorre a expulsão dos componentes voláteis do carvão e uma concentração de carbono.

Os valores médios de teores de cinza, carbono fixo e materiais voláteis obtidos para o carvão de endocarpo foram próximos aos observados por Boas et al. (2010), para endocarpo carbonizado

TABELA 3: Propriedades químicas e físicas do carvão vegetal produzido a partir da carbonização do epicarpo e endocarpo do fruto da macaúba.

TABLE 3: Chemical and physical properties of charcoal produced from the carbonization of the epicarp and endocarp of the macaw palm fruit.

\begin{tabular}{lcc}
\hline \multicolumn{1}{c}{ Parâmetros } & Epicarpo & Endocarpo \\
\hline Materiais voláteis (\%) & $18,29^{*}$ & 12,74 \\
Cinzas (\%) & $1,79^{*}$ & 3,84 \\
Carbono Fixo (\%) & $79,93^{*}$ & 83,43 \\
Densidade Aparente $\left(\mathrm{kg} / \mathrm{m}^{3}\right)$ & 1,024 & 1,007 \\
Poder Calorífico Superior $(\mathrm{Kcal} / \mathrm{kg})$ & $7464^{*}$ & 7859 \\
Densidade Energética $\left(\mathrm{GJ} / \mathrm{m}^{3}\right)$ & $31,99^{*}$ & 33,14 \\
\hline
\end{tabular}

Em que: Médias seguidas pelo asterisco na linha diferem entre si a 5\% de probabilidade pelo teste $\mathrm{F}$. 
a $550^{\circ} \mathrm{C}, 3,25,87,71$ e $9,04 \%$, respectivamente.

Quando comparado com resíduos de outras oleaginosas, os carvões de endocarpo e do epicarpo dos frutos da macaúba possuem vantagens em relação às suas propriedades químicas e físicas. Avaliando o carvão do epicarpo do pinhão manso e da torta das sementes, Vale et al. (2011) encontraram altos teores de cinzas 25,5 e $10,43 \%$, carbono fixo de 45,5 e $71,29 \%$, voláteis de 29 e $18,27 \%$ e baixo poder calorífico superior 3.954 e $6.233 \mathrm{Kcal} / \mathrm{kg}$ para o carvão do epicarpo e da torta do pinhão manso, respectivamente.

$\mathrm{Na}$ Figura 1 são apresentados os valores do balanço de massa do processo de carbonização do endocarpo e do epicarpo.

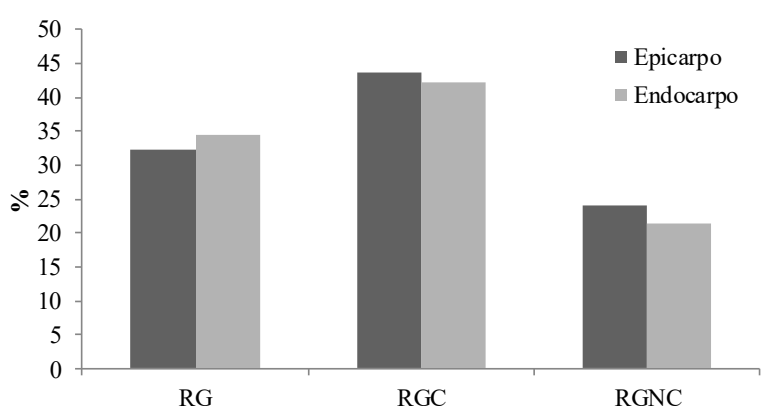

FIGURA 1: Rendimento gravimétrico (RG), rendimento de gases condensáveis (RGC) e rendimento de gases não condensáveis (RGNC) da carbonização do endocarpo e epicarpo oriundos do processamento dos frutos da macaúba.

FIGURE 1: Gravimetric yield (GY), condensable gases yield (CGY) and noncondensable gases yield (NCGY) of the endocarp and epicarp carbonization from the macaw palm fruit processing.

De acordo com a análise de variância, não houve efeito do tipo de resíduo da macaúba no rendimento gravimétrico em carvão vegetal, gases condensáveis e não condensáveis.

O RG da carbonização do endocarpo foi em média $34,35 \%$, enquanto do epicarpo foi de $32,31 \%$. Os RGC em média foram de 43,7 e $42,2 \%$ e os RGNC foram 23,99 e 21,32\% para a carbonização do epicarpo e endocarpo, respectivamente. Mesmo não tendo efeito significativo, o pequeno acréscimo do RG do carvão do endocarpo em relação ao do epicarpo é devido ao maior $\mathrm{CF}$ encontrado no carvão do endocarpo, este parâmetro contribui para um carvão de maior peso.
Boas et al. (2010) também encontraram valores similares no rendimento gravimétrico do carvão de endocarpo e caroço (endocarpo mais amêndoa) quando carbonizados a temperaturas de 550 e $650^{\circ} \mathrm{C}$. Em relação à produção de carvão oriunda de outras espécies, o carvão do epicarpo e do endocarpo também é satisfatório. Estes apresentam rendimentos gravimétricos, gases condensáveis e não condensáveis próximos aos obtidos com o carvão proveniente da madeira do eucalipto, conforme os estudos de Santos et al. (2011) e Neves et al. (2011).

\section{CONCLUSÕES}

De acordo com este estudo, todos os resíduos do processamento do fruto da macaúba têm potencial na geração de energia, especialmente o endocarpo e o epicarpo.

O carvão do endocarpo possui características adequadas para sua utilização como fonte de energia e como redutor.

A macaúba é uma espécie promissora para geração de biocombustíveis sólidos através dos seus frutos, por possuir diversos resíduos com boas características e alto valor energético.

\section{REFERÊNCIAS BIBLIOGRÁFICAS}

ASSOCIAÇÃO BRASILEIRA DE NORMAS TÉCNICAS. NBR 8112: Análise química imediata do carvão vegetal. Rio de Janeiro, 1986.

ASSOCIAÇÃO BRASILEIRA DE NORMAS TÉCNICAS. NBR 6923: Amostragem e preparação de amostras de carvão vegetal. Rio de Janeiro, 1981. ASSOCIAÇÃO BRASILEIRA DE NORMAS TÉCNICAS. NBR 8633: Carvão vegetal Determinação do poder calorífico - Método de ensaio. Rio de Janeiro, 1984

BOAS, M. A. V. et al. Efeito da temperatura de carbonização e dos resíduos de macaúba na produção de carvão vegetal. Scientia Forestalis, Piracicaba, v. 38, n. 87, p. 481-490, 2010.

CORTEZ, L. A. B.; LORA, E. S. Biomassa para energia. Campinas: Editora da Unicamp, 2008. $733 \mathrm{p}$.

EUROPEAN STANDARD NORME. EN 15103: Solid biofuels - Determination of bulk density. DIN EN 15103, Brussels 2010.

EMPRESA DE PESQUISA ENERGÉTICA. Balanço Energético Nacional 2011: Ano Base 2010. Rio de Janeiro: EPE, 2011. 266 p FUNDAÇÃO CENTRO TECNOLÓGICO DE MINAS GERAIS. Programa Energia. Produção 
de combustíveis líquidos a partir de óleos vegetais. Relatório final. Belo Horizonte: CETEC, 1983. p. 152.

ORGANIZAÇÃO DAS NAÇÕES UNIDAS PARA ALIMENTAÇÃO E AGRICULTURA. FAO Estatísticas 2011. Disponível em < http://faostat. fao.org/>. Acesso em: 6 maio 2013.

HIRSCHL, B. International renewable energy policy - between marginalization and initial approaches, Energy Policy, v. 37, n. 11, p. 4407-4416, 2009. LORENZI, H. et al. Palmeiras brasileiras e exóticas cultivadas. Nova Odessa: Instituto Platarum, 2004.

MIRANDA, I. P. A. et al. Frutos de Palmeiras da Amazônia. Manaus: MCT INPA, 2001.

NEVES, T. A. et al. Avaliação de clones de Eucalyptus em diferentes locais visando à produção de carvão vegetal. Pesquisa Florestal Brasileira, Colombo, v. 31, n 68, p.319-330, 2011.

PAULA, L. E. R. et al. Characterization of residues from plant biomass for use in energy generation. Cerne, Lavras, v. 17, n. 2, p. 237-246, 2011.

RAMOS, M. I. L. et al. Qualidade nutricional da polpa de bocaiúva Acrocomia aculeata (Jacq.) Lodd. Ciências Tecnologia de Alimentos, Campinas, v. 28, p. 90-94, 2008.
ROLIM, A. A. B. Óleos vegetais: usos gerais. Informe Agropecuário, Belo Horizonte, v. 7, n. 82, p. 17-22, 1981.

SANTOS, R. C. et al. Correlações entre os parâmetros de qualidade da madeira e do carvão vegetal de clones de eucalipto. Scientia Forestalis, Piracicaba v. 39, n. 90, p. 221-230, 2011.

SCARIOT, A.; LLERAS, E.; HAY, J. D. Flowering and fruiting phenologies of the palm Acrocomia aculeata: patterns and consequences. Biotropica, Malden - USA, v. 27, n. 2, p. 168-173, 1995.

Reproductive biology of the palm Acrocomia aculeata in Central Brazil. Biotropica, Malden USA, v. 23, n. 1, p. 12-22, 1991.

SILVA, J. N.; SOBRINHO, J. C.; SAIKI, E. T. Utilização de biomassa na secagem de produtos agrícolas via gaseificação com combustão adjacente dos gases produzidos. Engenharia Agrícola, Jaboticabal, v. 24, n. 2, p. 405-411, 2004.

VALE, A. T. D. et al. Potencial energético da biomassa e carvão vegetal do epicarpo e da torta do pinhão manso (Jatropha curcas). Cerne, Lavras, v. 17, n. 2, p. 267-273, 2011.

VITAL, B. R. Métodos de determinação da densidade da madeira. Viçosa: Sociedade de Investigações Florestais, 1984. 